\author{
Marquette University \\ e-Publications@Marquette
}

2016

\title{
Pilot Testing Behavior Therapy for Chronic Tic Disorders in Neurology and Developmental Pediatrics Clinics
}

\author{
Emily J. Ricketts \\ University of Wisconsin - Milwaukee \\ Donald L. Gilbert \\ Cincinnati Children's Hospital Medical Center \\ Samuel H. Zinner \\ Seattle Children's Hospital \\ Jonathan W. Mink \\ University of Rochester Medical Center \\ Tara D. Lipps \\ Cincinnati Children's Hospital Medical Center
}

See next page for additional authors

Follow this and additional works at: https://epublications.marquette.edu/psych_fac

Part of the Psychology Commons

\section{Recommended Citation}

Ricketts, Emily J.; Gilbert, Donald L.; Zinner, Samuel H.; Mink, Jonathan W.; Lipps, Tara D.; Wiegand, Geoffrey A.; Vierhile, Amy E.; Ely, Laura J.; Piacentini, John; Walkup, John T.; and Woods, Douglas W., "Pilot Testing Behavior Therapy for Chronic Tic Disorders in Neurology and Developmental Pediatrics Clinics" (2016). Psychology Faculty Research and Publications. 239.

https://epublications.marquette.edu/psych_fac/239 


\section{Authors}

Emily J. Ricketts, Donald L. Gilbert, Samuel H. Zinner, Jonathan W. Mink, Tara D. Lipps, Geoffrey A. Wiegand, Amy E. Vierhile, Laura J. Ely, John Piacentini, John T. Walkup, and Douglas W. Woods 
Marquette University

\section{e-Publications@Marquette}

\section{Psychology Faculty Research and Publications/ Psychology Department}

This paper is NOT THE PUBLI SHED VERSI ON; but the author's final, peerreviewed manuscript. The published version may be accessed by following the link in the citation below.

Journal of Child Neurology, Vol. 31, No. 4 (2016): 444-450. DOI. This article is @ SAGE Publications and permission has been granted for this version to appear in e-Publications@Marquette. SAGE Publications does not grant permission for this article to be further copied/distributed or hosted elsewhere without the express permission from SAGE Publications.
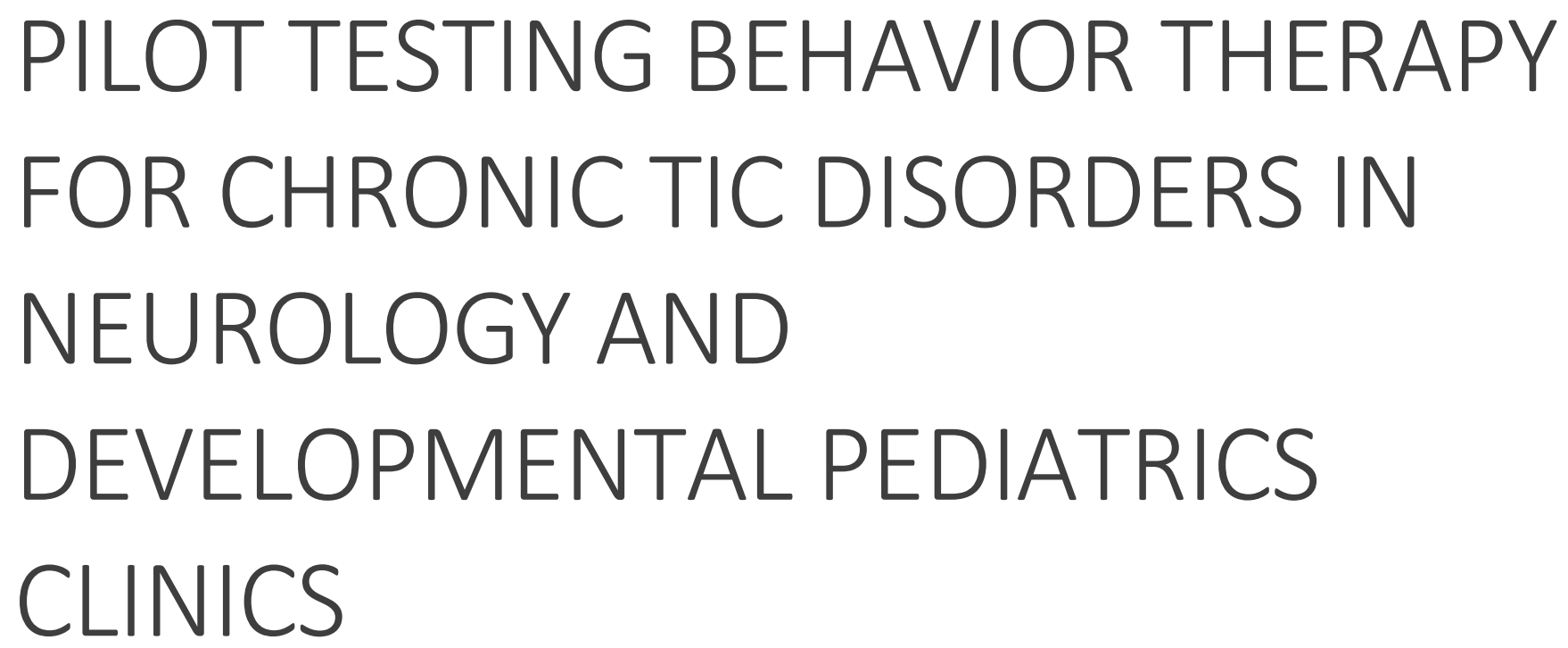

Emily J. Ricketts

University of California, Los Angeles, CA

Donald L. Gilbert

Cincinnati Children's Hospital Medical Center, Cincinnati, OH

Samuel H. Zinner

Seattle Children's Hospital, Seattle, WA

Jonathan W. Mink

University of Rochester Medical Center, Rochester, NY

Tara D. Lipps

Cincinnati Children's Hospital Medical Center, Cincinnati, $\mathrm{OH}$

Geoffrey A. Wiegand

Seattle Children's Hospital, Seattle, WA

Amy E. Vierhile 
University of Rochester Medical Center, Rochester, NY

Laura J. Ely

University of Wisconsin-Milwaukee, Milwaukee, WI

John Piacentini

University of Wisconsin-Milwaukee, Milwaukee, WI

John T. Walkup

Weill Cornell Medical College, White Plains, NY

Douglas W. Woods

Texas A\&M University, College Station, TX

\section{ABSTRACT}

Comprehensive Behavioral Intervention for Tics (CBIT) is an efficacious treatment with limited regional availability. As neurology and pediatric clinics are often the first point of therapeutic contact for individuals with tics, the present study assessed preliminary treatment response, acceptability, and feasibility of an abbreviated version, modified for child neurology and developmental pediatrics clinics. Fourteen youth (9-17) with Tourette disorder across 2 child neurology clinics and one developmental pediatrics clinic participated in a small case series. Clinician-rated tic severity (Yale Global Tic Severity Scale) decreased from pre- to posttreatment, $z=-2.0$, $\mathrm{P}<.05, \mathrm{r}=-.48$, as did tic-related impairment, $\mathrm{z}=-2.4, \mathrm{P}<.05, \mathrm{r}=-.57$. Five of the 9 completers (56\%) were classified as treatment responders. Satisfaction ratings were high, and therapeutic alliance ratings were moderately high. Results provide guidance for refinement of this modified CBIT protocol.

Keywords $\underline{\text { Tourette disorder, } \underline{\text { tics, }} \text {, behavior therapy, dissemination, neurology, pediatrics }}$

Chronic tic disorders, including Tourette disorder, are characterized by tics: sudden, repetitive movements and/or sounds. ${ }^{-}$Chronic tic disorders are associated with psychosocial, family, academic, and physical impairment, $\underline{2} \underline{3}$ prompting many to seek treatment. ${ }^{4}$ Pharmacologic interventions are commonly prescribed but have variable, often incomplete efficacy and may lead to intolerable side effects. ${ }^{5}$ Behavior therapy, particularly Comprehensive Behavioral Intervention for Tics (CBIT), is also efficacious in children and adults. ${ }^{6}-\underline{8}$ More recently, CBIT/Habit Reversal Training (HRT) has been endorsed as a first-line treatment for chronic tic disorders. $\underline{-}-\underline{11}$

CBIT, including HRT, function-based intervention, relaxation training, and behavioral rewards, $\underline{12}$ was designed for use by mental health professionals. Despite demonstrated efficacy and dissemination efforts, CBIT is not widely available. ${ }^{4}$ A recent survey found behavior therapy for children with Tourette disorder is often not sought because parents did not know where it could be accessed. ${ }^{4}$ Compounding the problem, children with chronic tic disorders are typically first seen in primary care clinics and referred to psychiatry or pediatric neurology specialists, where the probability of finding CBIT-trained therapists is low. ${ }^{4}$

One possible solution is to train nontherapist health care providers to deliver CBIT in settings where patients with tics are initially referred. Therefore we sought to (1) develop a modified CBIT protocol for use in child neurology and developmental pediatrics clinics (CBIT-NP), and (2) conduct a small case series to examine initial treatment response, acceptability, and feasibility. 


\section{METHOD}

\section{MANUAL DEVELOPMENT}

CBIT $\underline{12}$ includes eight 60 - to 90-minute sessions held weekly or every other week over a 10-week period. CBIT blends HRT (awareness training, competing response [CR] training, and social support), function-based assessment and intervention, relaxation techniques, and behavioral rewards. Awareness training involves sensitizing the patient to detect occurrences of tics and any accompanying premonitory urges preceding tics. Next, the patient learns to perform a behavior that is physically incompatible with the tic (ie, $C R$ ) in response to premonitory urge or tic occurrence, for 1 minute or until the urge diminishes. Social support involves enlisting a person (usually a child's parent or other caregiver) to encourage and remind the patient to use the CR by praising and prompting correct implementation. With HRT, the patient's tics are listed, and treated cumulatively, with target tic selection based on priority of subjective intolerability, adding approximately one tic per week. Function-based assessment and intervention involves identifying internal or environmental factors (occurring before or after tics) associated with tic exacerbation, and suggesting modifications to these factors. Relaxation techniques include diaphragmatic breathing and progressive muscle relaxation to relieve stress and muscle tension. Behavioral rewards are used to motivate treatment engagement.

The original protocol was modified using recommendations for adapting empirically supported treatments to primary care settings. $\frac{13}{}$ Initially, the directors and health care providers at 3 study sites, including 2 child neurology clinics (University of Rochester Medical Center and Cincinnati Children's Hospital Medical Center) and 1 developmental pediatrics clinic (Seattle Children's Hospital [ $\mathrm{SCH}]$ ), were surveyed about practice parameters and preferences (ie, typical visit duration, time available for CBIT-NP, preferences for session frequency and duration, potential treatment barriers, and treatment modifications).

Time allotted for clinic follow-up visits ranged from 20 to 40 minutes. The maximum duration that could be allotted for CBIT-NP sessions ranged from 15 to 30 minutes, and the ideal session number ranged from 4 to 8 . A lack of available support staff and time demands were cited as potential treatment barriers. Suggested CBIT-NP modifications included simplifying functional assessment procedures and conducting treatment independently from standard clinic visits. Results were used to modify the CBIT manual and workbook를 for use in the participating clinics.

\section{PARTICIPANTS}

Seventeen patients were screened for eligibility, with 14 enrolled. See Table 1 for participant characteristics. 
Table I. Baweline Characteristes.

\begin{tabular}{|c|c|c|c|c|}
\hline \multirow{2}{*}{$\begin{array}{l}\text { Characteristic } \\
\text { Study Center, n (s) }\end{array}$} & \multicolumn{2}{|c|}{$\begin{array}{l}\text { All participants } \\
\qquad(\mathrm{n}=14)\end{array}$} & \multicolumn{2}{|c|}{$\begin{array}{l}\text { Completers } \\
(\mathrm{n}=9)\end{array}$} \\
\hline & & & & \\
\hline Sestele Children's Hoxpial & 6 & $(42.9)$ & 5 & $(55,6)$ \\
\hline $\begin{array}{l}\text { Cineinnati Children's Hospital } \\
\text { Medical Center }\end{array}$ & 4 & $28.6)$ & 2 & $(22.2)$ \\
\hline $\begin{array}{l}\text { University of Rochester Medical } \\
\text { Center }\end{array}$ & 4 & $28.6)$ & 2 & $(22.2)$ \\
\hline \multicolumn{5}{|l|}{ Patent demographies } \\
\hline Age mean (SD) & 12.2 & $(2.2)$ & 11.9 & (24) \\
\hline $\begin{array}{l}\text { K.BIT/2 IQ standard score, mean } \\
\text { (SD) }\end{array}$ & $1 \mid 4.2$ & (II.I) & || 14.6 & $(13.0)$ \\
\hline Male gonder. $n(\mathrm{n})$ & 10 & {$[7,4]$} & 6 & $(667)$ \\
\hline \multicolumn{5}{|l|}{ Ethnicity n $(3)$} \\
\hline Non-Hispanic & 14 & $(100)$ & 9 & (100) \\
\hline \multicolumn{5}{|l|}{ Race, in ( } \\
\hline Cuucasin & 9 & (64.3) & 6 & $(66.7)$ \\
\hline Arien American & I & $(7,1)$ & 0 & (0) \\
\hline Astan & 2 & $(14,3)$ & 1 & $(I 1, i)$ \\
\hline $\begin{array}{l}\text { Native Hwailan or other Pacific } \\
\text { lshunder }\end{array}$ & 1 & $(7.1)$ & 1 & $(11.1)$ \\
\hline $\begin{array}{l}\text { Binchal (African-American: } \\
\text { Cuucapan) }\end{array}$ & 1 & $(7,1)$ & 1 & $(11,1)$ \\
\hline $\begin{array}{l}\text { On tic-reduction madiction at stitry } \\
n[\%]\end{array}$ & 3 & {$[21.4)$} & 4 & $(44.4)$ \\
\hline Twopparent household, n (5) & 9 & $(69.2)$ & 7 & $(778)$ \\
\hline \multicolumn{5}{|l|}{ Parents" hyhert education, n (M) } \\
\hline Partal hid whool & 1 & $(7,7)$ & 0 & (0) \\
\hline Colege graduate & 2 & $(15.4)$ & 1 & $(11.1)$ \\
\hline Professional deyree & 10 & $(76.9)$ & 8 & $(68.9)$ \\
\hline \multicolumn{5}{|l|}{ Dagnoses, in (\%) } \\
\hline Tourette disorder & 14 & $(100)$ & 9 & (100) \\
\hline $\begin{array}{l}\text { Atention-defisis hypenceivity } \\
\text { disorder (ADHD) }\end{array}$ & 3 & $(21,4)$ & I & $(1, i, 1)$ \\
\hline Obessivecompultive disorder & I & $(7.1)$ & I & (II.I) \\
\hline Generalized andiety disorder & I & $(7,1)$ & 0 & (0) \\
\hline Postenumatic stress disorder & 1 & $(7,1)$ & 0 & (100) \\
\hline \multicolumn{5}{|l|}{ Yale Global Tie Strerity Seale, mean (SD) } \\
\hline Total kare & 24.8 & (6.9) & 25.4 & (7.5) \\
\hline Mator substale & I4.I & $(4.1)$ & 14.4 & $(3.0)$ \\
\hline Phonik subscale & 10.6 & $(4.8)$ & 10,9 & $(5,6)$ \\
\hline Impaiment score & 20.0 & $(8,2)$ & 1800 & (9.3) \\
\hline $\begin{array}{l}\text { Clinieal Giobal lmpresion-Sentity } \\
\text { sole kore, mean (5D) }\end{array}$ & 4.1 & $(0.8)$ & 4.0 & $(0.9)$ \\
\hline
\end{tabular}

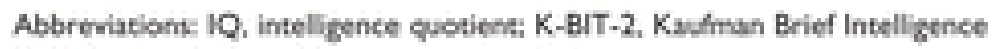
Tex-Second Edition: SD. wanderd dewation

\section{PROCEDURE}

Recruitment took place primarily through clinic patient flow. Patients who appeared eligible were invited to participate. Parents completed institutional review board-approved consent forms (children provided assent), and families were screened for eligibility by independent evaluators. Inclusion criteria included $(a)$ age 9 to 17, (b) chronic tic disorder or Tourette disorder diagnosis, $\underline{\underline{14}}$ (c) Clinical Global Impressions-Severity scale score $\underline{\underline{15}} \geq 4$ 
(moderately ill or worse), (d) Yale Global Tic Severity Scale (YGTSS) ${ }^{16}$ Total Score $\geq 14,(e)$ unmedicated or stable on psychotropic medication for $\geq 6$ weeks with no planned changes during study participation, and $(f)$ fluency in English. Exclusion criteria included $(a)$ composite intelligence quotient $(\mathrm{IQ})<80$ on the Kaufman Brief Intelligence Test-2nd Edition, ${ }^{17}$ (b) Substance Abuse or Dependence, or Conduct Disorder diagnosis within the past 3 months, $(c)$ lifetime Pervasive Developmental Disorder, Mania, or Psychotic Disorder diagnosis, $(d)$ any serious condition requiring treatment not provided in the study, and $(e)$ previous HRT for tics.

At the initial screen, the independent evaluator assessed patient IQ, and Yale Global Tic Severity Scale and Clinical Global Impressions-Severity scores. Parents provided patient demographic, and medical/psychiatric information. The independent evaluator re-administered the Yale Global Tic Severity Scale and re-rated the Clinical Global Impressions-Severity score 7 to 10 days later. Those no longer meeting criteria were excluded. The 6-session CBIT-NP protocol was then initiated. At the posttreatment assessment, occurring within 1 week of the final session, the independent evaluator reassessed tic and global severity, and rated global improvement using the Clinical Global Impressions-Improvement scale. Additionally, patients rated the therapeutic alliance, and families rated treatment satisfaction and provided feedback on treatment utility.

\section{MEASURES}

\section{KAUfMAN BRIEF INTELLIGENCE TEST-SECOND EDITION}

The Kaufman Brief Intelligence Test-Second Edition ${ }^{17}$ is a well-validated intelligence test for patients aged 4 to $90, \underline{\underline{18}}$ yielding verbal, nonverbal, and composite IQ scores.

\section{Yale Global Tic SeVerity SCAle}

The Yale Global Tic Severity Scale $\frac{16}{6}$ is a semistructured interview producing independent severity ratings for motor and vocal tics, combining to produce a 0- to 50-point total tic severity scale. Additionally, the Yale Global Tic Severity Scale includes an independent 0- to 50-point tic-related impairment scale. $\underline{16, \underline{19}}$

\section{CLINICAL GLOBAL IMPRESSION-SEVERITY AND IMPROVEMENT SCALES}

The Clinical Global Impressions-Severity $\underline{15}$ is a subjective measure of patient global illness severity rated on a 1-7 Likert-type scale, with higher scores reflecting greater severity. The Clinical Global Impressions-Improvement Scale ${ }^{15}$ is a 1-7 measure of global improvement since baseline. Scores of "very much improved" (1) or "much improved" (2) indicate positive treatment response. These scales display high internal consistency, and interrater reliability, good to high concurrent validity, and sensitivity to change. $\underline{20}-\underline{22}$

\section{Client SATISFACTION QuestionNAIRE}

The Client Satisfaction Questionnaire ${ }^{23}$ is an 8-item, 4-point measure of satisfaction with health services. Higher scores indicate greater satisfaction. The questionnaire has high internal consistency, $\underline{23, \underline{24}}$ good concurrent validity, $\underline{\underline{25}}$ and excellent test-retest reliability. $\underline{\underline{26}}$

\section{USABILITY FORM}

Usability forms assessed understanding and perceptions of treatment procedures at posttreatment.

\section{THERAPIST FIDELITY FORM}

Treatment sessions were videotaped and assessed for fidelity. Treatment experts rated 10 randomly selected sessions on a 1-4 ( 1 = poor, 2 = moderate, 3 = good, 4 = excellent) scale, with higher ratings indicating greater adherence. 
Children's Hospital]) underwent cross-site training before conducting assessments. Independent evaluators were supervised by the on-site investigators, each with extensive experience in administering the study measures. Prior to study initiation, independent evaluators were expected to score within $15 \%$ of an expert rater on the Yale Global Tic Severity Scale, and within 1 point on the Clinical Global Impressions scales.

\section{CBIT-NP PROVIDER TRAINING AND SUPERVISION}

Treatment was provided by nurse practitioners with child neurology expertise (University of Rochester Medical Center, Cincinnati Children's Hospital Medical Center), or a physician specializing in developmental and behavioral pediatrics (Seattle Children's Hospital). Training was multifaceted and designed to maximize treatment fidelity. First, clinicians reviewed the CBIT-NP manual and readings on behavioral treatment of tic disorders. Second, they watched a video-recorded CBIT training $\underline{\underline{Z}}$ and were required to pass (minimum $90 \%$ ) a knowledge test about the treatment protocol. Finally, they attended a live, intensive training, conducted by the principal investigator, consisting of a detailed review of the manual, observation of standard CBIT treatment session videos, and supervised role-play of key treatment techniques. To guard against treatment drift, the principal investigator provided follow-up case consultation as needed via phone or email throughout the study.

\section{DESCRIPTION OF CBIT-NP}

CBIT-NP consisted of 6 sessions, delivered over 6 to 8 weeks. Session 1 lasted 25 minutes, with subsequent sessions lasting 20 minutes. One week prior to treatment, parents received a workbook and a supplemental DVD. Sessions were conducted with the child and parent present, excepting older adolescents (ie, aged 16-17).

Prior to session 1: The parent and child completed a detailed list of current tics, and read about psychoeducational information, environmental influences on tics, and self-monitoring of tics at home to prepare for the first session.

Session 1: The clinician described the treatment rationale, and allotted time for questions about psychoeducational material. The clinician reviewed the precompleted tic list and asked the child to demonstrate each tic and select the first one to be treated. An explanation of the potential environmental impact on tics was provided. Parental guidelines for a tic-management environment were discussed, including (1) not reacting (ie, not responding with positive or negative attention) to the child's tics beyond neutral prompts and praise of CR use, (2) not expressing frustrations with the child's condition when within earshot, (3) interacting with the child about his or her strengths rather than being the "tic police," (4) expecting as much from the child as one would if he or she did not have tics, (5) keeping the child mentally and physically engaged, (6) having a consistent bedtime routine, (7) ensuring the child gets adequate sleep, and (8) reminding the child to relax when he or she feels stressed. Additionally, tic monitoring was reviewed, and session 2 assignments (ie, parent- and self-monitoring of the first tic, HRT portion of the DVD, creating a tic signal [ie, antecedent behaviors and pre-tic "urges"] list, and implementing environmental changes) were given.

Session 2: The tic list was updated, tic monitoring was discussed, and implementation of environmental changes was reviewed. HRT was conducted for tic 1. For Awareness Training, the clinician and patient reviewed the rationale and the tic signals list. The patient practiced noticing the tic and tic signals. For CR Training, the clinician provided a rationale for CR use, selected a CR for tic 1 , demonstrated the CR for about 5 seconds, and checked for patient understanding. The patient practiced the CR a few times for about 10 seconds each time; and implemented the CR for 1 minute (or until the urge subsided) contingent upon urge or tic occurrence, as the tics and/or urges occurred within session. If the tic did not happen in session, the child was asked to mimic a tic and use the CR for 1 minute. Lastly, social support involved identifying a parent and instructing him/her to read in the workbook about providing effective social support. Assignments included CR practice, monitoring of tic 2, watching the DVD for guidance on CRs, and continuing implementation of tic-management environment suggestions. Sessions 3 through 5: These sessions were structured similarly, with the clinician conducting HRT generally for one tic per week (advancing from most to least bothersome). Following session 3 , patients 
also read about relaxed breathing, watched a DVD demonstration, and practiced relaxing during targeted situations with the aid of a worksheet. In sessions 4 through 6 , the clinician received feedback from the patient regarding use of relaxed breathing, and discussed specific situations in which relaxation techniques may help the patient relax in future situations. Clinician demonstrations or patient observations of relaxed breathing were only performed if the patient reported difficulties with implementation.

Session 6: Treatment was reviewed, HRT was conducted for any remaining tics, and relapse prevention was discussed.

\section{RESULTS}

Of the 14 enrolled participants, 13 received treatment. Overall, 5 (36\%) withdrew from the study before completing the posttreatment assessment (igure 1). Of the 9 study completers, 7 (78\%) received 6 sessions, 1 $(11 \%)$ received 5 , and $1(11 \%)$ received 4.

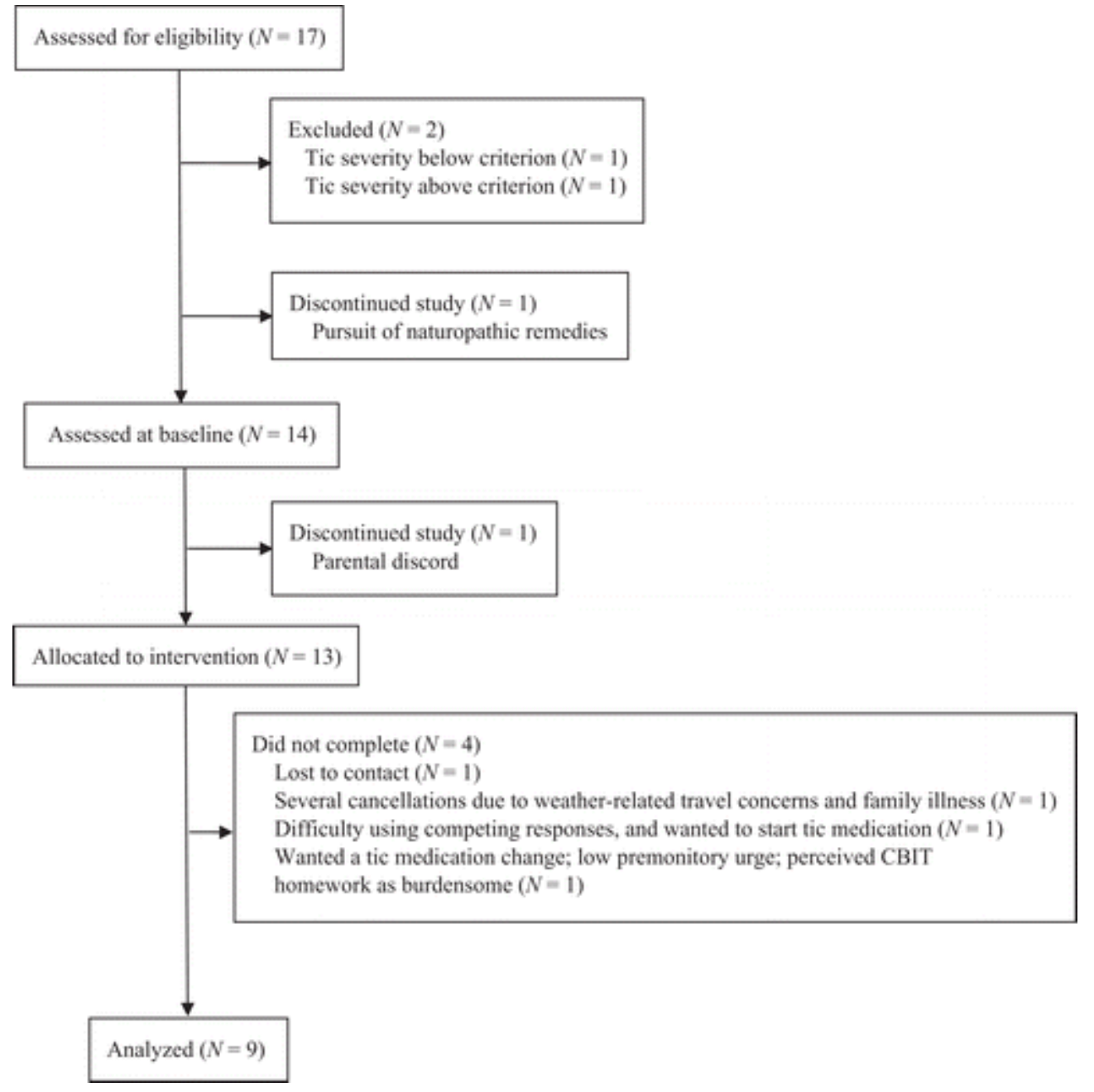

Figure 1. Flow of participants through the trial.

The Wilcoxon signed-rank test was used to assess pre- to posttreatment changes in tic symptoms (Table 2). Nine participants were included in primary analyses. The Yale Global Tic Severity Scale total tic severity significantly decreased ( $\underline{\text { Table } 2}$ ) from pre- (median $=23.0)$ to posttreatment (median $=20.0), z=-2.0, p<.05, r=-.48(2$ tailed). There was also a significant decrease in Yale Global Tic Severity Scale impairment from pre- (median = 
20.0) to posttreatment (median $=0.0), z=-2.4, p<.05, r=-.57$ (2-tailed). Six of the 9 completers reported a Yale Global Tic Severity Scale impairment score of zero at posttreatment.

\begin{tabular}{|c|c|c|c|c|c|c|c|}
\hline \multirow[b]{2}{*}{ Measure } & \multicolumn{2}{|c|}{ Pretreatment } & \multicolumn{2}{|c|}{ Posttreatment } & \multirow[b]{2}{*}{$z$ score } & \multirow[b]{2}{*}{ P value } & \multirow[b]{2}{*}{$t$ value } \\
\hline & Mean (SD) & Median (QQR) & Mean (SD) & Median (IQR) & & & \\
\hline \multicolumn{8}{|l|}{ YGTSS } \\
\hline Total score & $25.4(7.5)$ & $23.0(14.0)$ & $20.9(8.7)$ & $20.0(13.5)$ & -2.0 & .04 & -.48 \\
\hline Motor subscale & $14.4(3.0)$ & $14.0(5.5)$ & $12.1(4.1)$ & $12.0(6.0)$ & -1.7 & .09 & -.40 \\
\hline Phonic subscale & $10.9(5.6)$ & $9.0(10.0)$ & $8.8(4.8)$ & $10.0(7.0)$ & -1.3 & .21 &.,- 30 \\
\hline Impairment score & $18.9(9,3)$ & $20.0(10.0)$ & $7.8(10.9)$ & $0.0(15.0)$ & -2.4 & .02 & -.57 \\
\hline CGI.S & $4.0(3.7)$ & $4.0(20)$ & $2.3(1.1)$ & $2.0(2.0)$ & -2.6 & .01 & -.60 \\
\hline
\end{tabular}

Abbreviadions: CGI.S. Clinical Global Impressions-Severity Scale; SO, standard deviation; YGTSS, Yale Global Tic Severity Scale; YGTSS Tocal and Impairment scores are independene ratings.

Global impairment, as rated by the Clinical Global Impressions-Severity scale (Table 2) also decreased from pre( median $=4.0$ ) to posttreatment (median $=2.0), z=-2.6, p<.05, r=-.60$ (2-tailed). At the final assessment, 5 of the 9 completers (56\%) were classified as treatment responders, meaning they had a score of "very much improved" or "much improved" on the Clinical Global Impressions-Improvement scale.

At posttreatment, patient $($ mean $=26.0$, standard deviation $=4.8$; scale range $=8-32$ ) and parent $($ mean $=30.9$, standard deviation $=1.4$ ) satisfaction ratings for the 9 study completers were high. The mean adherence rating for videotaped treatment sessions was 3.2 (on a scale from 1 to 4, with 4 indicating perfect adherence; standard deviation $=0.8$ ), indicating good treatment fidelity. Experts co-rated $25 \%$ randomly selected Yale Global Tic Severity Scale assessments. On average, independent evaluator and expert ratings differed by $11.6 \%$ (standard deviation $=8.1 \%$ ), within the standard of $15 \%$ of the expert rating.

\section{CLINICIAN PERCEPTIONS OF FEASIBILITY}

Clinicians completed a posttreatment survey regarding perceived CBIT-NP feasibility. Perceptions were that 20minute sessions were mostly feasible to administer once experience with the intervention had been obtained, but longer sessions were preferred. Clinicians reported that treatment length (ie, 6 sessions) was appropriate, but suggested that as few as 4 sessions may suffice for patients who grasp treatment techniques quickly. All clinicians reported that incorporation of CBIT-NP into their clinics was feasible but anticipated several logistical barriers, including time, limited clinic space, late patient arrivals, families with several treatment questions raised at the end of sessions, interruptions (ie, pager, being on call), the need for interpreter services for nonEnglish-speaking patients, documentation, billing, and insurance reimbursement issues, poor patient attendance, and attrition.

\section{DISCUSSION}

In the present study, $\mathrm{CBIT} \stackrel{12}{2}$ was modified for use in neurology and developmental pediatrics settings, and the preliminary treatment response, acceptability, and feasibility of this new version (CBIT-NP) were tested. Results showed significant reductions in tic severity, tic-related impairment, and global severity. Of note, 6 of the 9 treatment completers were rated as having no tic-related impairment at posttreatment. Likewise, the treatment response rate was $56 \%$ for study completers and $36 \%$ for those assessed only at baseline (eg, intent to treat). This compares to the $57 \%$ completer and $53 \%$ intent-to-treat response rates from the large, randomized controlled efficacy trial for standard CBIT protocol- which was conducted in mental health settings using behavioral psychologists as therapists. Reasons for the intent-to-treat difference in outcomes across the 2 studies are unclear, but may include the lower treatment intensity (fewer and shorter sessions) of CBIT-NP, which decreased the depth of content and time allotted for HRT skills practice within sessions, while increasing between-session assignments; and the relative lesser expertise in CBIT-NP among the medical professionals providing this treatment compared to the therapists in the original trial. ${ }^{?}$ 
Importantly, treatment adherence ratings were good (mean $=3.2$ on a 1 to 4 scale), with $80 \%$ of sessions rated "good" or "excellent." This is comparable to results of the initial trial, in which $88 \%$ of sessions were rated "good" or higher, $-\underline{7}$ suggesting that training medical professionals to provide quality behavioral treatment is feasible.

With respect to the broader feasibility of implementing CBIT-NP, several issues arose that should be addressed in future treatment revisions. First, although CBIT-NP was designed to be brief and highly structured to accommodate time constraints, clinician time burden remained a barrier to implementation across sites. Second, elements of session structure with respect to content, duration, and number were key issues. Identifying a realistic session structure that allows for completion of goals in an efficient manner will be important in future treatment adaptations. Third, scheduling appointment times that were convenient for both patients and clinic staff posed a challenge largely due to physical space limitations. Considering that both specialty clinical and primary care settings have historically been designed to meet the needs of a large number of patients presenting on an infrequent basis, $\underline{27}$ this challenge is not surprising. However, space limitations would likely resolve over time should behavioral services be integrated into these clinical settings on a long-term basis. $\underline{28}$ Fourth, poor treatment adherence was an issue in some cases. It is unclear whether this was due to low motivation, homework burden, or other factors. Homework nonadherence is a commonly cited barrier across treatment types and settings $\frac{29}{}$ but may pose a greater barrier in brief therapy settings in which practitioners must place a greater emphasis on out-of-session assignments. Furthermore, the homework burden may have been greater relative to the original manual, as increased home preparation aided the brief session format.

There are notable study limitations. The sample size was small, and no control group was used. Additionally, independent evaluators were not blinded to pre-posttreatment status or study hypotheses, which may have influenced clinical ratings at posttreatment. Furthermore, the $36 \%$ attrition rate is considerably higher than in the original CBIT trial. Although high child- and parent-reported satisfaction ratings were reported, the elevated drop-out rate may be indicative of lower treatment acceptability. With respect to reasons for treatment dropout, at least 2 of 5 subjects withdrew due to reported CBIT-NP-related challenges (ie, lack of premonitory urge, difficulty using CRs, and perceived between-session "homework" burden) and/or a desire to start or change ticreduction medication. Another subject was lost to contact for unknown reasons, whereas 2 withdrew for reasons unrelated to study procedures (eg, parental discord, family illness, travel difficulties). Beyond these reasons, it is possible that youth receiving CBIT in medical settings may have different expectations regarding the treatment and work it entails relative to patients treated in mental health settings. Perhaps the addition of motivational interviewing to assess and elicit readiness for behavioral treatment, and increased psychoeducation addressing potential treatment challenges would improve retention. Furthermore, no followup assessment was included; thus, it is unclear whether treatment effects are lasting. Future research will require larger sample sizes, controlled study designs, and short- and long-term follow-up to evaluate maintenance of treatment gains.

The successful integration of CBIT into neurology and pediatric settings has several important clinical implications. First and perhaps most importantly, this will greatly increase the availability of CBIT. Second, because children with tic disorders most often initially present in medical settings, they will have the opportunity to receive treatment earlier in the course of the disorder, which may not only positively impact treatment outcomes but also reduce the individual and overall morbidity of these disorders. Finally, implementation of CBIT in medical settings will significantly broaden patient treatment options and potentially reduce medication exposure to youth with milder forms of tic disorder. However, future research addressing the impact of CBIT-NP on prescription practices, and comparing the feasibility of delivering CBIT-NP independent of, versus blended with, standard clinic visits, is needed to fully realize the potential benefits of this intervention. 


\section{AUTHOR Contributions}

EJR contributed to study coordination, data collection, manuscript drafting and editing, statistical analysis, and interpretation of data. DLG contributed to study supervision, manuscript editing, and interpretation of data. SHZ contributed to study supervision, data collection, manuscript editing, and interpretation of data. JWM contributed to study supervision, manuscript editing, and interpretation of data. TDL contributed to data collection, and manuscript editing. GAW contributed to data collection, and manuscript editing. AEV contributed to data collection, and manuscript editing. LJE contributed to manuscript editing, and interpretation of data. JP contributed to obtaining funding, study concept and design, manuscript editing, and interpretation of data. JTW contributed to obtaining funding, study concept and design, manuscript editing, and interpretation of data. DWW is the corresponding author, and contributed to obtaining funding, study concept and design, supervision, manuscript drafting and editing, and interpretation of data.

\section{DECLARATION OF CONFLICTING INTERESTS}

The authors declared the following potential conflicts of interest with respect to the research, authorship, and/or publication of this article: EJR, TDL, GAW, AEV, and LJE report no disclosures. DLG has received honoraria from the Tourette Syndrome Association and Centers for Disease Control and Prevention (CDC) and the American Academy of Pediatrics; serves on the medical advisory board for the Tourette Syndrome Association; and has received book royalties from Elsevier. He has received research support (for Tourette syndrome, ADHD) from the NIH (NIMH R01 MH092520, NIMH R01 MH081854), from the Cincinnati Children's Hospital Research Foundation, Otsuka Pharmaceuticals (clinical trial, Tourette syndrome), Ecopipam Pharmaceuticals (clinical trial, Tourette syndrome), and AstraZeneca (clinical trial, Tourette syndrome). SHZ receives honoraria for continuing education presentations sponsored by the Tourette Syndrome Association through their collaborative partnership with the CDC, and receives grant support from NIMH. JWM serves on an independent Data and Safety Monitoring Board for Edison Pharmaceuticals, performs Consultant work for Medtronic, Inc., and receives honoraria for serving as Associate Editor of Neurology for the American Academy of Neurology. JP receives grant support from NIMH, the Pettit Foundation and Pfizer Pharmaceuticals, book royalties from Guilford Press and Oxford University Press, and speaking honoraria from the Tourette Syndrome Association and International OCD Foundation. JTW receives royalties from Oxford University Press for treatment manuals on tic disorders, honoraria for continuing education presentations from the Tourette Syndrome Association, and royalties from Guilford Press for a book on Tourette disorder. He also receives consulting fees from Eli Lilly and JAZZ Pharmaceuticals and lecture fees from CMP Media, Medical Education Reviews, McMahon Group, DiMedix, and the Tourette Syndrome Association. He receives free drug and matching placebo from Pfizer and Lilly, and free drugs from Abbott for NIMH-funded clinical trials. He reports receiving fees for consultation with defense counsel and submission of written reports in litigation involving GlaxoSmithKline. DWW receives royalties from the Guilford Press, Oxford University Press, and Springer Press, and honoraria for presentations sponsored by the Tourette Syndrome Association through their collaborative partnership with the CDCP.

\section{FUNDING}

The authors disclosed receipt of the following financial support for the research, authorship, and/or publication of this article: The research reported in this manuscript was supported by a grant to DWW, JP, and JTW, with subcontracts to DLG, JWM, and SHZ from the Tourette Syndrome Association. The content is the responsibility of the authors and is not necessarily representative of the views of the Tourette Syndrome Association.

\section{ETHICAL APPROVAL}

The study was approved by the institutional review boards at the coordinating institution (University of Wisconsin-Milwaukee Institutional Review Board no. 10.218) and 3 treatment sites (Seattle Children's Hospital/University of Washington Institutional Review Board Protocol no. 13308, Cincinnati Children's Hospital Medical Center Institutional Review Board no. CCHMC 2010-0924, and University of Rochester Medical Center 
Research Subjects Review Board no. RSRB00032932). Institutional review board-approved informed consent and child assent were obtained from all participants.

\section{REFERENCES}

1. American Psychiatric Association. Diagnostic and Statistical Manual of Mental Disorders. 5th ed. Washington, DC: American Psychiatric Association; 2013. Google Scholar, Crossref

2. Conelea, CA, Woods, DW, Zinner, SH. Exploring the impact of chronic tic disorders on youth: results from the Tourette Syndrome Impact Survey. Child Psychiatry Hum Dev. 2011;42:219-242. Google Scholar, Crossref, Medline

3. Storch, EA, Merlo, LJ, Lack, C. Quality of life in youth with Tourette's syndrome and chronic tic disorder. J Clin Child Adolesc Psychol. 2007;36:217-227. Google Scholar, Crossref, Medline

4. Woods, DW, Conelea, CA, Himle, MB. Behavior therapy for Tourette's disorder: utilization in a community sample and an emerging area of practice for psychologists. Prof Psychol Res Pr. 2010;41:518-525. Google $\underline{\text { Scholar, }}$ Crossref

5. Qasaymeh, MM, Mink, JW. New treatments for tic disorders. Curr Treat Options Neurol. 2006;8:465-473. Google Scholar, Crossref, Medline

6. Cook, CR, Blacher, J. Evidence-based psychosocial treatments for tic disorders. Clin Psychol Sci Pract. 2007;14:252-267. Google Scholar, Crossref

7. Piacentini, J, Woods, DW, Scahill, L. Behavior therapy for children with Tourette disorder. JAMA. 2010;303:1929-1937. Google Scholar, Crossref, Medline

8. Wilhelm, S, Peterson, AL, Piacentini, J. Randomized trial of behavior therapy for adults with Tourette syndrome. Arch Gen Psychiatry. 2012;69:795-803. Google Scholar, Crossref, Medline

9. Verdellen, C, van de Griendt, J, Hartmann, A, Murphy, T. European clinical guidelines for Tourette syndrome and other tic disorders. Part III: Behavioural and psychosocial interventions. Eur Child Adolesc Psychiatry. 2011;20:197-207. Google Scholar, Crossref, Medline

10. Steeves, T, McKinlay, BD, Gorman, D. Canadian guidelines for the evidence-based treatment of tic disorders: behavioural therapy, deep brain stimulation, and transcranial magnetic stimulation. Can J Psychiatry. 2012;57:144-151. Google Scholar, Link

11. Murphy, TK, Lewin, AB, Storch, EA, Stock, S. Practice parameter for the assessment and treatment of children and adolescents with tic disorder. J Am Acad Child Adolesc Psychiatry. 2013;52:1341-1359. Google $\underline{\text { Scholar, }}$ Crossref, Medline

12. Woods, DW, Piacentini, J, Chang, S. Managing Tourette Syndrome: A Behavioral Intervention for Children and Adults Therapist Guide: A Behavioral Intervention for Children and Adults Therapist Guide. New York: Oxford University Press; 2008. Google Scholar, Crossref 
13. Robinson, P. Adapting empirically supported treatments to the primary care setting: a template for success. In: O'Donohue, WT, Byrd, MR, Cummings, NA, Henderson, DA, eds. Behavioral Integrative Care: Treatments That Work in the Primary Care Setting. New York: Brunner-Routledge; 2005:53-71. Google Scholar

14. American Psychiatric Association. Diagnostic and Statistical Manual of Mental Disorders. 4th ed. Washington, DC: American Psychiatric Association; 2000. Google Scholar

15. Guy, W. ECDEU Assessment Manual for Psychopharmacology-Revised. Rockville: DHEW Publication Number ADM 76-338. US Department of Health, Education, and Welfare, Public Health Service, Alcohol, Drug Abuse, and Mental Health Administration, NIMH Psychopharmacology Research Branch, Division of Extramural Research Programs; 1976:223-244. Google Scholar

16. Leckman, JF, Riddle, MA, Hardin, MT. The Yale Global Tic Severity Scale: initial testing of a clinician-rated scale of tic severity. J Am Acad Child Adolesc Psychiatry. 1989;28:566-573. Google Scholar, Crossref, Medline

17. Kaufman, AS, Kaufman, NL. Kaufman Brief Intelligence Test-Second Edition (KBIT-2). Circle Pines: American Guidance Service; 2004. Google Scholar

18. Bain, SK, Jaspers, KE. Review of Kaufman Brief Intelligence Test, Second Edition. J Psychoeduc Assess. 2010;28:167-174. Google Scholar, Link

19. Storch, EA, Murphy, TK, Geffken, GR. Reliability and validity of the Yale Global Tic Severity Scale. Psychol Assessment. 2005;17:486-491. Google Scholar, Crossref, Medline

20. Haro, JM, Kamath, SA, Ochoa, S. The Clinical Global Impression-Schizophrenia scale: A simple instrument to measure the diversity of symptoms present in schizophrenia. Acta Psychiatrica Scandinavica. 2003;107: s16-s23. Google Scholar, Crossref

21. Khan, A, Khan, SR, Shankles, EB, Polissar, NL. Relative sensitivity of the Montgomery-Asberg Depression rating scale, the Hamilton Depression rating scale and the Clinical Global Impressions rating scale in antidepressant clinical trials. Int Clin Psychopharmacol. 2002;17:281-285. Google Scholar, Crossref, Medline

22. Zaider, TI, Heimberg, RG, Fresco, DM. Evaluation of the Clinical Global Impression Scale among individuals with social anxiety disorder. Psychol Med. 2003;33:611-622. Google Scholar, Crossref, Medline

23. Larsen, DL, Attkisson, C, Hargreaves, WA, Nguyen, TD. Assessment of client/patient satisfaction: development of a general scale. Eval Program Plann. 1979;2:197-207. Google Scholar, Crossref, Medline

24. Nguyen, TD, Attkisson, CC, Stegner, BL. Assessment of patient satisfaction: development and refinement of a Service Evaluation Questionnaire. Eval Program Plann. 1983;6:299-314. Google Scholar, Crossref, Medline

25. Barker, DA, Orrell, MW. The Psychiatric Care Satisfaction Questionnaire: a reliability and validity study. Social Psychiatry Psychiatr Epidemiol. 1999;34:111-116. Google Scholar, Crossref, Medline

26. Roberts, RE, Attkisson, CC, Mendias, RM. Assessing the Client Satisfaction Questionnaire in English and Spanish. Hispanic J Behav Sci. 1984;6:385-395. Google Scholar, Link 
27. Bodenheimer, T. Primary care-will it survive? N Engl J Med. 2006;355:861-864. Google Scholar, Crossref, Medline

28. Scharf, DM, Eberhart, NK, Schmidt, N. Integrating primary care into community behavioral health settings: Programs and Early Implementation Experiences. Psychiatr Serv. 2013;64:660-665. Google Scholar,

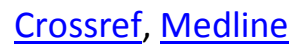

29. Kazantzis, N, Shinkfield, G. Conceptualizing patient barriers to nonadherence with homework assignments. Cogn Behav Pract. 2007;14:317-324. Google Scholar, Crossref 